\title{
Lecciones y desafíos para la investigación científica en la Estación Atacama UC_Oasis de niebla Alto Patache. Conversaciones sobre desierto y niebla con Horacio Larraín y Pilar Cereceda
}

\author{
Juan Luis García’
}

\begin{abstract}
JLG: Este año se cumplen 20 años de investigación en la Estación Atacama UC _ Oasis de niebla Alto Patache ¿Cuáles son los principales aprendizajes obtenidos, y qué ustedes destacarían, a lo largo de todos estos años?
\end{abstract}

HL: Creo que se ha aprendido muchísimo, tanto acerca del comportamiento de la niebla misma, como de sus efectos sobre el ecosistema desértico y, también, sobre sus ricas potencialidades como recurso hídrico limpio para el futuro de la presencia humana en la zona costera. Por ejemplo, se ha aprendido la relación entre velocidad del viento y el monto de captación de agua de niebla; la importancia de la altitud sobre el nivel del mar, el valor de la gran proximidad de la línea de costa, la importancia de la presencia de "puntas" o estribaciones de montaña rocosa que penetran mar adentro, hacia el Oeste, interrumpiendo el aspecto rectilíneo de ésta y generando, en el océano, surgencias marinas; sobre el efecto e importancia de los "portezuelos", en las partes altas de la cadena montañosa costera; $y$, por fin, la importancia de la existencia de sectores altos que miran directamente al Sur o Suroeste, enfrentando directamente los vientos predominantes.

PC: Coincido con todo lo que Horacio narra en su respuesta. Dentro de mis aprendizajes también destacaría el formar un Centro como el CDA con una sede en Alto Patache, es una tarea titánica, que requiere la participación de todas las comunidades: la de nuestro Instituto de Geografía UC, las autoridades de la Casa Central UC y colegas. La mayor experiencia es haber logrado incentivar a la comunidad científica nacional y extranjera, pero es una labor que se debe alimentar permanentemente, porque si no se hace, ésta se pierde y los miembros del CDA quedamos solos.

JLG: ¿Cuál es la importancia que tiene la experimentación en la propia Estación Atacama UC como método para adquirir todas estas nociones y enseñanzas?

HL/PC: Muy importante, por cierto. Por ejemplo, se aprendió, gracias a experimentos ad hoc, las altitudes ideales de captación del agua de la niebla, poniendo aparatos pequeños, idénticos, entre los $350 \mathrm{~m}$ y los $850 \mathrm{~m}$ de altitud, en un transecto Oeste-Este. De similar manera, se aprendió, con mediciones en terreno, que sólo en ciertos sectores de la costa y no en cualquier parte, se puede producir captación de niebla en forma significativa. Es decir, allí donde se dan los factores geográficos decisivos para la creación de "oasis de niebla". Por otro lado, la investigación en la estación sobre los tipos y diseños de instrumentos captadores, sus deficiencias y fortalezas, en especial en referencia a la resistencia contra el viento, la capacidad de condensación de mallas, 
y la resistencia de materiales contra la humedad ambiente, es un constante aprehender de esta tecnología y su aplicación en el desierto de Atacama.

\section{JLG: Horacio, tú mencionas que Alto Patache es un "oasis de niebla", ¿qué relevancia posee y han poseído este tipo de áreas para la habitabilidad humana en el desierto de Atacama?}

HL: Múltiples, por cierto. Las principales y que llaman la atención son respecto de la capacidad de sobrevivencia de especies de plantas xerófitas; sobre la relación íntima entre presencia de vegetación y fauna terrestre asociada en el oasis; y sobre la relación íntima entre vegetación, fauna y presencia humana como depredadora y consumidora de especies vegetales y animales terrestres (arqueología). En este sentido, estimo que se ha ganado para la arqueología costera una más rica y profunda comprensión sobre la existencia de importantes yacimientos arqueológicos en áreas que constituyen o constituyeron "oasis de niebla". La presencia y actividad humana del pasado, por parte de pescadores y recolectores, se percibe mucho más claramente gracias a la presencia de estos oasis. Un "oasis de niebla" significa nuevos y potentes recursos para el pescador, los que le permiten ampliar considerablemente su dieta alimenticia. Esto se ha detectado claramente en lugares de Arica, Iquique, y Antofagasta (Punta Pichalo, Alto Patache, Chipana, Punta Gruesa Iquique-, Mamilla, Cobija, etc). Estamos empezando a dimensionar la relación entre el "Fenómeno de El Niño" y la presencia de oasis costeros. Así mismo, hay gran potencial de campo de estudio con ayuda de los biólogos marinos, la correlación existente entre "puntas" que sobresalen y puntos de "surgencias" de aguas marinas profundas, creadoras de alta productividad de algas, peces y fauna marina concomitante, enfrentando los oasis de neblina terrestres.

JLG: Hablemos un poco de historia, ¿cómo descubren Alto Patache? ¿qué tenía/tiene de especial este sector del desierto de Atacama?

HL: El sitio del "oasis de niebla" era ya bien conocido por los biólogos de la Universidad Arturo Prat (por ejemplo, por Walter Sielfeld). A mi llegada a la Universidad Arturo Prat en lquique (marzo de 1993), me contacté muy pronto con Walter, quien había sido buen amigo del entomólogo Luis E. Peña Guzmán, hermano de mi mujer, Marta. Por ahí comenzó el acercamiento. Como yo me interesaba, también especialmente, por la Entomología (por mi antigua amistad con Luis Peña desde el año 1953), y sabiendo Walter de este interés, en una conversación en el año 1995 o 1996, me aconsejó ir a conocer el sitio. El objetivo de la visita sería colectar insectos. Al efecto me hizo un detallado croquis para poder llegar. A fines del año 1996 (cuyo dato exacto debe estar en mis Diarios de Campo), subimos en mi Ford Escort al lugar, logrando salvar la difícil repechada de la cuesta. Seguimos la huella abierta hacía muy poco tiempo por la empresa eléctrica que instaló las torres de alta tensión para llevar electricidad producida en el puerto de Patache a la zona minera cordillerana. Una vez arriba, lo primero que encontramos fueron rastros de arqueología (raspadores y lascas de sílex) y de presencia de guanacos (revolcaderos y sus típicos senderos). Siendo yo arqueólogo, mi interés por la arqueología se despertó en seguida: ¡había rastros de presencia humana arriba!

Recorrimos la zona y hallamos, bajo plantas, los primeros insectos (coleópteros). Quedamos encantados, pues el lugar ofrecía de todo: arqueología, entomología, botánica y de refilón, presencia de niebla abundante. 
PC: Nuevamente coincido con Horacio. Para mí Alto Patache reúne todas las condiciones para que especialistas de todas las áreas temáticas de un desierto absoluto puedan trabajar allí. Desde la arqueología, geografía, botánica, zoológica, sociología, antropología hasta la tecnología de captación de niebla y de la energía solar, eólica, etc. Quizás lo más importante que logramos fue juntar una gran diversidad de científicos nacionales y extranjeros, siendo la que más me llegó al alma, el trabajo de los arquitectos paisajistas y los artistas de landart. También los escritores y poetas. Es un lugar mágico que encantó a las comunidades costeras que Horacio llevó a conocer y que nunca pensaron que tenían un paraíso a sus espaldas.

JLG: Según tengo entendido, en los años que reconocieron el potencial de Alto Patache, ya existían científicos que trabajaban sobre la camanchaca en el Norte Grande, ¿cómo fue la relación e integración con ellos?

HL: Mis contactos en Antofagasta, en la Universidad del Norte, con los estudios de la camanchaca costera que llevaban a cabo en aquellos años (1960-64) el profesor Carlos Espinosa Arancibia, profesor de física, y otros investigadores de dicha Universidad (en un período en que yo mismo pertenecí a dicha Universidad (1963-1965), me permitieron conocer y visitar varias veces lugares de niebla; como por ejemplo, Cerro Moreno, Los Nidos, Mina Andrómeda y El Portezuelo, que eran visitados por los físicos. En su compañía, pudimos probar instrumentos de captación fabricados por el profesor Espinosa siguiendo el modelo clásico de Grunov. Espinosa pronto patentará otros diseños de captación, usando como malla la arpillera usada en la fabricación de sacos. Fue mi temprano contacto con la camanchaca de Antofagasta y los primeros esfuerzos de captación en la Universidad del Norte. Yo tenía por entonces 34 años.

Apenas conocí el lugar de Alto Patache (1996) y me di cuenta de sus enormes potencialidades de estudio en el ámbito geográfico, di aviso a Pilar Cereceda Troncoso, geógrafa del Instituto de Geografía de la Pontificia Universidad Católica de Chile, con quien habíamos ya trabajado varios años en las alturas de El Tofo, investigando la camanchaca. Nos concertamos y un día de fines de 1996 (¿tal vez septiembre u octubre?) Pilar llegó a lquique acompañado del joven profesor Pablo Osses a reconocer el lugar. Quedaron encantados y ahí mismo se decidió presentar, próximamente, un Proyecto FONDECYT para el estudio de la niebla en el lugar. Fue nuestro primer Proyecto. Teníamos como apoyo y experiencia, nuestros años de trabajos en los cerros de El Tofo, cosechando agua de niebla (1984-1989, aproximadamente). Por tanto, no comenzábamos de cero. Y disponíamos del generoso apoyo del profesor Carlos Espinosa de Antofagasta, quien nos ofreció de inmediato toda su ayuda y nos envió copia de todos sus trabajos en dicha especialidad.

JLG: ¿Cuáles fueron las primeras investigaciones que se realizaron y cómo se gestó el grupo de estudios del Desierto de Atacama, cuya continuidad hoy está representado por el Centro UC desierto de Atacama (CDA)?

HL: El detalle debería obtenerse de los diversos Proyectos consecutivos que se presentaron, tanto a FONDECYT como a otras instituciones, con cuyo concurso fue posible realizar las numerosas tareas del trabajo de campo. Lo primero que interesó saber fue qué posibilidades concretas de captación existían (montos de captación), para lo cual se probó a diversas altitudes y con un tipo común de instrumento mediante el SFC (Standard Fog Collector) de $1 \mathrm{~m}^{2}$ de malla raschel, de un tipo especial (romboidal), instalado a 2 metros del suelo. Sin embargo, para participar en 
los Congresos de Niebla, que se hacían en distintas partes del mundo, los investigadores también realizaron estudios sobre flora autóctona, arqueología y sistemas de captación.

Hasta donde yo recuerdo, la idea de crear un grupo de estudios de largo plazo en el lugar, surgió para evitar considerar el sitio, como un momentáneo o esporádico lugar de experiencias, que se podía abandonar en cualquier momento, terminado un Proyecto. Siempre yo tuve la idea de solicitar a Bienes Nacionales, la concesión del sitio por un largo período de tiempo, para realizar experiencias en las que pudieran participar los alumnos del Instituto de Geografía de la UC y también de otras Universidades invitadas. Porque siempre tuve en mente, la intención de dar agua a las caletas más cercanas a Alto Patache, concretamente a la caleta de Chanabaya. Lo que requería, de necesidad, que existiera una institución patrocinante, dueña o concesionaria del lugar con nieblas; y en este caso, la Pontificia Universidad Católica de Chile podía cumplir ese rol. Insistí mucho ante Pilar en la idea de obtener una concesión del oasis por parte de Bienes Nacionales. Y efectivamente, se solicitó la concesión y finalmente, después de un largo proceso, se obtuvo. Los Rectores de la UC, primero el Dr. Pedro Pablo Rosso y el Dr. Ignacio Sánchez, después, siempre se mostraron muy entusiasmados con la idea y visitaron el lugar, animándonos a continuar con nuestro trabajo y esfuerzo.

A partir de este grupo interdisciplinario de estudios en Alto Patache, surgió la idea de continuar como Centro UC Desierto de Atacama (CDA); entidad que, en la actualidad, potencia aunar y congregar las investigaciones realizadas por investigadores de la UC en zonas desérticas del país, no solo en Alto Patache, sino también en el Norte Chico.

JLG: Claramente este aspecto sigue muy vigente en la dirección del CDA y constituye uno de sus mayores desafíos, el integrar la investigación del desierto y formular avances interdisciplinarios, de su conocimiento. Al respecto, ¿cuál es su apreciación sobre la evolución del uso e investigación en Alto Patache?

HL: En el período en que Pilar Cereceda fue Jefa de los Proyectos en el sector (yo siempre fui el encargado y responsable del sitio, no más), se procuró entusiasmar y atraer a numerosos otros científicos a estudiar el área, bajo muy diversos aspectos. Así, llegaron alemanes, israelitas, estadounidenses, canadienses, españoles, etc., interesados en diversos aspectos. Muchas tesis de alumnos de la UC fueron realizadas aquí, como también algunas tesis de alumnos de arqueología de la Universidad Bolivariana de lquique, donde yo mismo fui profesor por varios años. Mi impresión es que debiera existir una cuidada planificación general, de largo plazo, de lo que se puede o se debe investigar en la zona de aquí al año 2050 (por decir una fecha lejana); es relevante siempre privilegiar programas bien estructurados, de largo plazo. A mi entender, urge hacerlo hacia el futuro. Esto es vital para darle proyección a la estación y al CDA en este lugar.

JLG: Estoy muy de acuerdo con la idea de tener programas bien estructurados de largo plazo como mencionas Horacio. De hecho, creo que sin una hoja de ruta la actividad se puede volver ineficiente y perder gran parte de su potencial. Desde hace algunos años construimos un modelo de sistemas denominado el "Geo_Ecosistema de la Costa de Atacama", cuya estructura multidisciplinar nos ha permitido converger con colegas de varias disciplinas para entender el funcionamiento del ambiente de neblinas costeras y su dinámica temporal. Esa es nuestra hoja de ruta actual en este tema. 
PC: En mi parecer, también rescato que lo más importante en sus inicios fue el trabajo pionero que se hizo en relación al comportamiento de la niebla y el gran número de tesis de alumnos de la UC, de otras universidades chilenas y del extranjero; incluso varias fueron de doctorados. Pero, si debo hacer un mea culpa, creo que fallamos en incorporarlos más como equipos interregionales e interdisciplinarios.

Sin embargo, el mayor éxito fue el gran número de científicos extranjeros que fueron al lugar en diversas oportunidades. Siempre estuvimos a sus órdenes, los acompañamos e hicimos proyectos conjuntos; trajeron sus instrumentos, sus alumnos, escribimos papers como autores y coautores. Incluso llegamos a exponer en el Nevada Museum of Art y donde dejamos para sus archivos, materiales de Arquitectura y Geografía para las generaciones futuras. También estuvimos presente en "Documenta 13", una de las más prestigiadas exposiciones de arte y medioambiente del mundo. En las Bienales internacionales, tuvimos presencia de alto nivel, recibiendo premios, así como en congresos internacionales de niebla, donde tuvimos participación como miembros del comité organizador en varias de ellas; fuimos sede en La Serena de la $4^{a}$ Conferencia Internacional de Niebla con más de 100 participantes de todos los continentes.

Desde entonces creo que la Estación Atacama UC ha evolucionado exitosamente, con gran número de papers de científicos nacionales e internacionales; con la formación de grupos de la UC donde Ingeniería lideró equipos de investigación tecnológica de atrapanieblas, con prototipos ensayados en el Norte Chico; además de equipos de energía solar con equipos de medición instalados en la estación para evaluar esta componente. En Geografía, se ha logrado avanzar fuertemente en la aplicación de sensores remotos, lo que actualmente sigue relacionado con el Centro Latinoamericano de la Universidad de Heidelberg, con doctorandos y alumnos de Magister.

La mayor innovación de los últimos años fue haber consolidado un Centro de Acción en la Estación Atacama UC en base a domos con agua potable de niebla y energía solar, con todas las comodidades para hacer clases, alojar investigadores y alumnos que investigan en diversas disciplinas. Esta iniciativa permite traer más investigadores de todo el país y de otros de universidades de alto nivel internacional.

\section{JLG: Por último, ¿cómo visualizan el futuro de Alto Patache? ¿cuál(es) es(son) su(s) poten- cial(idades) y los desafíos que esperan aún ser resueltos?}

HL: Un primer aspecto que se me viene a la mente y que me ha acompañado muchos años, es la factibilidad de entregar agua potable producida por la nube, de alta calidad, a las caletas costeras, y que nunca se ha podido concretar por razones de diversa índole. Su importancia, sin embargo, para el futuro de las caletas costeras, es indudable y debería ser un punto a considerar muy seriamente en futuros proyectos. La experiencia de Chungungo (Región de Coquimbo) es elocuente en este sentido y entrega muchas lecciones. Requiere, eso sí, el concurso de avezados antropólogos para un adecuado trato con las comunidades.

Por otro lado, yo he escrito, años atrás, un documento que está en poder de la UC, sobre la creación de lo que he llamado un "Parque geo-ecológico de agua de niebla", instalado en la terraza marina de los 50-60 m de altitud, exactamente bajo el oasis de niebla Alto Patache y alimentado por éste (mediante captadores en altura) de agua potable y electricidad (obtenida del viento). 
Fue y sigue siendo mi gran sueño. Tal vez pueda servir de pauta para planificar investigaciones futuras en el área, que involucren también el área baja, de la terraza marina, que es parte de la Concesión por Bienes Nacionales. Es una zona amplia y subutilizada en la actualidad, a pesar de que existen allí al menos tres muy interesantes sitios arqueológicos, de campamento de pescadores y recolectores marinos muy antiguos. Uno fue motivo de un trabajo nuestro en la revista "Polis" de la Universidad Bolivariana, con el descubrimiento de entierros de pescadores de la época Chinchorro (5.000-6.000 A.C.). Pienso que allí, a la vista y junto a la carretera, se podría mostrar al público una instalación de investigación y de museología de la niebla y el resultado de sus investigaciones a través del mundo. Como hay mucha arqueología en el lugar, se podrían agregar muestrarios arqueológicos de los antiguos ocupantes del oasis. El lugar sería, a la vez, Museo (se muestran todos los instrumentos de captación ideados en el mundo y arqueología, lugar de estudio con biblioteca especializada sobre la niebla y laboratorios. Anexo se hallaría el Centro de Experimentación Agrícola con agua de niebla para la formación de los hijos de los pescadores de las caletas vecinas. Habría pues, dos áreas bien aprovechadas en la actual Concesión de Patache: la de arriba (actual a $780 \mathrm{~m}$ s.n.m.), donde se demostraría dónde y cómo se produce el agua de la niebla (con alojamiento en los domos existentes hoy) y abajo, en la terraza marina (a los 50-60 m s.n.m.), con la creación de una gran estructura tanto educacional (granja agrícola para pescadores) como museológica (de formato doble: Museo de la Neblina y Museo Arqueológico).

Sintetizando, me atrevería a decir que el futuro, parcialmente, podría consistir en:

a) Mostrar al gran público en qué consiste concretamente la investigación en esta temática, mediante la creación del Centro autosuficiente (en materia energética y agua potable) tal como ya he señalado;

b) Y arriba, en el oasis mismo, mostrar, en el borde del acantilado rocoso, "jardines" de plantas nativas endémicas del oasis, regadas con agua de niebla constantemente, mediante captadores en altura (ya se hizo en nuestro tiempo una mini experiencia en este sentido con notable resultado de crecimiento y floración de plantas);

c) Organización de sistemas de visitas guiadas de estudiantes e interesados a ambos sectores (Colegios, Universidades), para promocionar la utilización del agua de niebla en el futuro de esta desértica región de Chile.

JLG: Esto aparece como un proyecto de alto interés tanto regional como nacional. Concuerdo que deberíamos apuntar en esa dirección y evaluar la factibilidad de tal magnífica idea y su consecuente impacto. Sería un justo traspaso a la comunidad de todos estos años de investigación en el área de Alto Patache, sobre todo pensando en la labor pública de la universidad.

PC: Además de lo dicho por Horacio Larraín, soy una gran optimista, ya que Rectoría UC, y la Facultad de Historia, Geografía y Ciencia Política se han involucrado y apoyado al CDA en forma muy especial. Asimismo, Geografía ha tomado el desafío de hacer del CDA y Patache una sede de la UC en el Norte Grande, donde se cumple la misión de descentralizar la universidad y llegar desde el Norte Grande hasta la Patagonia.

El potencial mayor es poner a disposición de los científicos locales y alumnos de diversas universidades del país, para que puedan contar con un lugar para hacer investigación de alto nivel, con las comodidades que se requiere, por ejemplo, contar con energía para computadores, internet, celular y también la infraestructura necesaria para poder vivir en un ambiente agradable, y 
que durante los periodos de niebla, se pueda contar con las comodidades para descansar, dormir y tener agua para una buena ducha caliente al regreso de las campañas y poder disfrutar de una buena reunión de camaradería con los colegas y alumnos.

En relación a los desafíos a resolver, la idea de hacer un lugar de conservación como lo expuesto por el Dr. Larraín, un Geoparque, es primordial, ya que podría tener apoyo gubernamental, siendo parte del SNASPE, y contar con financiamiento del Estado y de las entradas que debieran cobrarse por utilizar las instalaciones y el material recopilado a lo largo de los años. 
